\title{
Influence of Relative Length of Baffles on the Flow Field in a Rectangular Enclosure: A Computational Study
}

\author{
B H Lakshmana Gowda* and N Gopi ${ }^{\dagger}$
}

\section{Abstract}

The flow field in an enclosure with length to width ratio (L/B) of 2 and varying number of baffle plates (n) is computationally investigated. For each value of $n$ the influence of the relative length of the baffle plate (ratio of length of the plate a to the width of the enclosure B, a/B ratio) on the flow field is investigated. Mainly the influence on the flow pattern is brought out. It is seen that by controlling the $\mathrm{a} / \mathrm{B}$ ratio, the regions of recirculation, separation and stagnant zones can be controlled.

Keywords: Baffles, Computations, Flow patterns

\section{Nomenclature}

a Length of the baffle

B Width of the chamber

b Spacing between baffles

L Length of the chamber

$l_{1} \quad$ Length of inlet

\footnotetext{
* Department of Mechanical Engineering, BTL Institute of Technology, Bommasandra, Bengaluru-560099, India, bhlgowda@yahoo.com

† Department of Mechanical Engineering, BTL Institute of Technology, Bommasandra, Bengaluru-560 099, India.
} 
$1_{2} \quad$ Length of outlet

n Number of baffles

U Free stream velocity

W Inlet/ Outlet width of the enclosure

\section{Introduction}

Rectangular enclosures with baffle plates are found in many applications in industries and is the most common geometry used in heat exchangers in nuclear power plants. The baffles act as passive elements to enhance the heat transfer between hot and cold fluids. In shell-and-tube heat exchangers, the cross flow through the enclosure is obtained by means of baffle plates, responsible for changing the direction of flow and for increasing the heat exchange time between fluid and the heated surfaces. Baffles are also used in contact tanks in water treatment plants and for drying processes. In general, baffles increase the turbulence levels and thus the heat exchange ratios. A very complex flow field results, where besides turbulence, boundary layer separation and recirculation zones occur [1-4]. A comprehensive and systematic study of the influence of all the parameters involved on the effective performance of the baffles is needed. A study is underway to investigate these aspects both experimentally and using computations. In this paper the results of the computational studies carried out on a rectangular enclosure with length to width ratio (L/B, Figure1) of 2 with

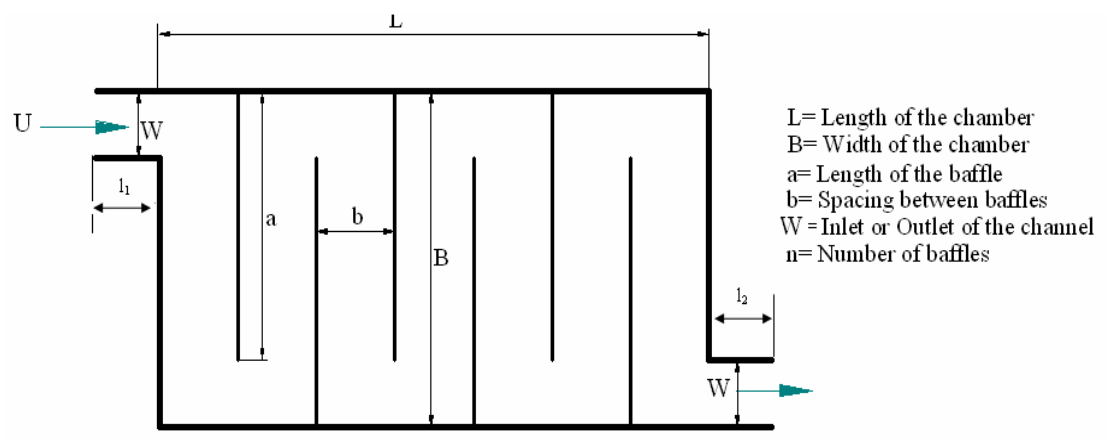

Figure 1: Configuration considered 
various number of baffle plates (n) are presented. For each value of $\mathrm{n}$, the relative length of the baffle plate (a) to the width of the enclosure (B) is varied. The flow patterns obtained for the several cases are compared and the influence of a/B brought out.

The configuration considered in this study is shown in Figure 1. The flow is from left to right as indicated and the various parameters which can influence the flow field are: the length and width ratio $L / B$, the ratio of the length of the baffle to the width of enclosure $a / B$, the number of baffle plates $n$, the entry widths at inlet and outlet $W$, the spacing $b$ between the baffles, the lengths at the entry and exit $l_{1}$ and $l_{2}$ respectively, and the Reynolds number. In the present study, $\mathrm{L}=300 \mathrm{~mm}$ and $\mathrm{B}=150 \mathrm{~mm}$ i.e., $\mathrm{L} / \mathrm{B}=2, \mathrm{~W}=$ $l_{1}=l_{2}=30 \mathrm{~mm}, \mathrm{n}=2,4,6$ and 8 are considered. At each value of $\mathrm{n}$, results are obtained for a/ $\mathrm{B}$ ratios of $0.8,0.7,0.6$ and 0.5 . The main aim has been to obtain the variation of the flow patterns with $\mathrm{a} / \mathrm{B}$ ratio.

\section{Computations}

Computations are carried out using the CFD code FLUENT, Version 12 employing the finite volume method. The results are obtained for $\mathrm{L} / \mathrm{B}=2$ (Figure 1 ) for $\mathrm{n}=2,4,6$ and 8 . At each value of $\mathrm{n}$, computations are carried out for $\mathrm{a} / \mathrm{B}=0.8,0.7,0.6$ and 0.5 for a given Reynolds number. The geometry and the meshing (with triangular elements) are generated by using 'Workbench' in the CFD code. The 'grid independency check' is carried out and based on the results, a total of 406583 nodes are employed for the solution. After prescribing the necessary boundary conditions, the cold flow analysis has been carried out. Turbulent intensity and hydraulic diameter are specified as the initial conditions for the inlet turbulence. Realizable k- $\varepsilon$ model is used for turbulence. SIMPLE algorithm has been used for pressure-velocity coupling to solve the steady state governing equations. All the results are obtained at a Reynolds number of 6000 referred to the entry width $(\mathrm{W}=0.03 \mathrm{~m})$ and velocity at entry $(\mathrm{U}=0.19 \mathrm{~m} / \mathrm{s})$. 


\section{Results}

As mentioned earlier, computations have been carried out for the case with $\mathrm{L}=300 \mathrm{~mm}$ and $\mathrm{B}=150 \mathrm{~mm}$ i.e., $\mathrm{L} / \mathrm{B}=2, \mathrm{~W}=\mathrm{l}_{1}=\mathrm{l}_{2}=30$ $\mathrm{mm}, \mathrm{n}=2,4,6$ and 8 and at each value of $\mathrm{n}$, for $\mathrm{a} / \mathrm{B}=0.8,0.7,0.6$ and 0.5 . The velocity vector plot for each case is obtained and presented. The vector lengths are so chosen that these plots in effect show the flow patterns.

In Fig. 2 is shown the results for $\mathrm{L} / \mathrm{B}=2, \mathrm{n}=6$ and $\mathrm{a} / \mathrm{B}=0.8$ to a larger scale compared to those presented in Figs. 3 to 6 to bring out the details more clearly. However, discussion of the results are presented with reference to Figs. 3 to 6 as comparisons can obtained from these figures.

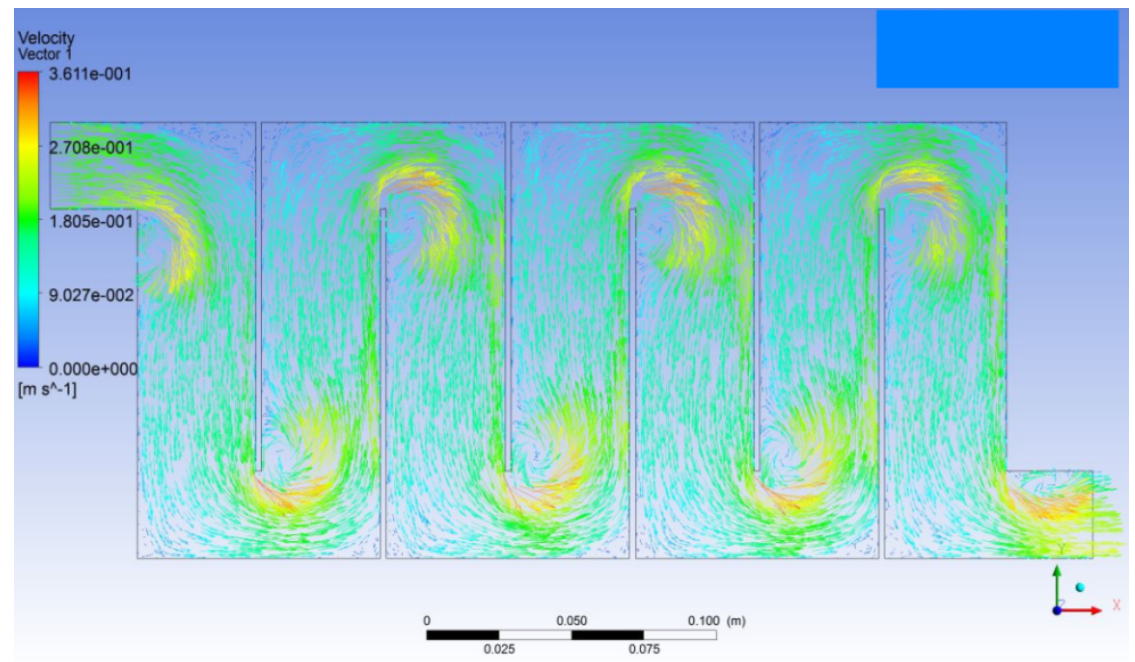

Figure 2: Velocity Vectors: $\mathrm{L} / \mathrm{B}=2, \mathrm{n}=6, \mathrm{a} / \mathrm{B}=0.8$ 


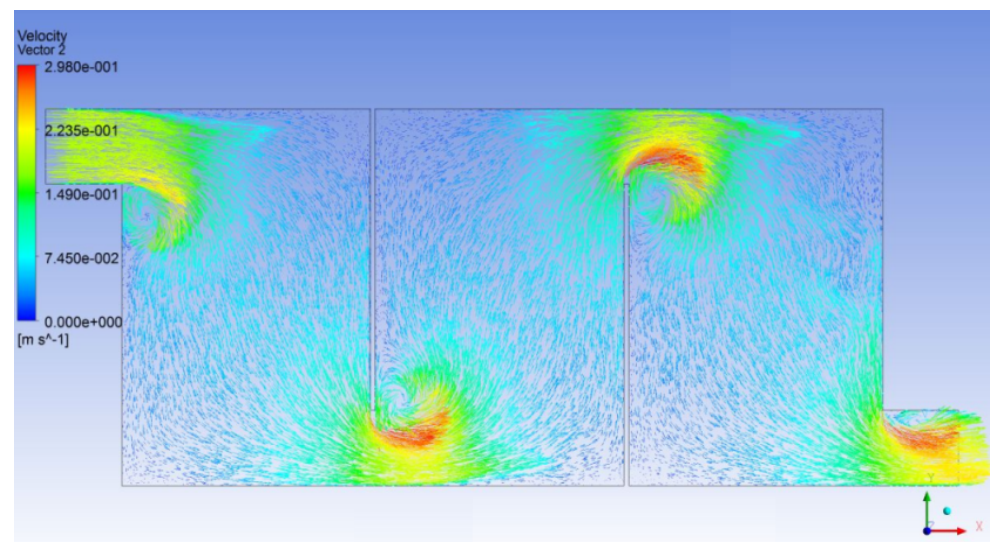

a/B

a) 0.8

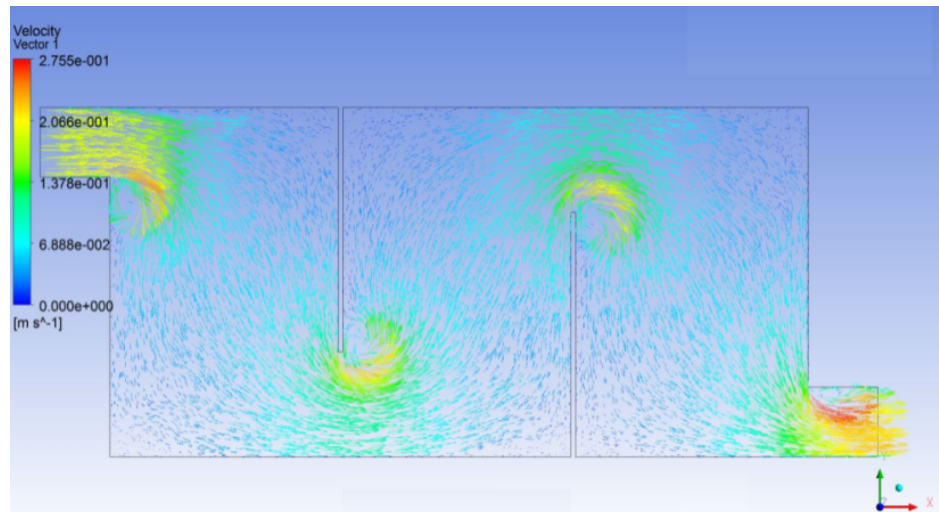

b) 0.7

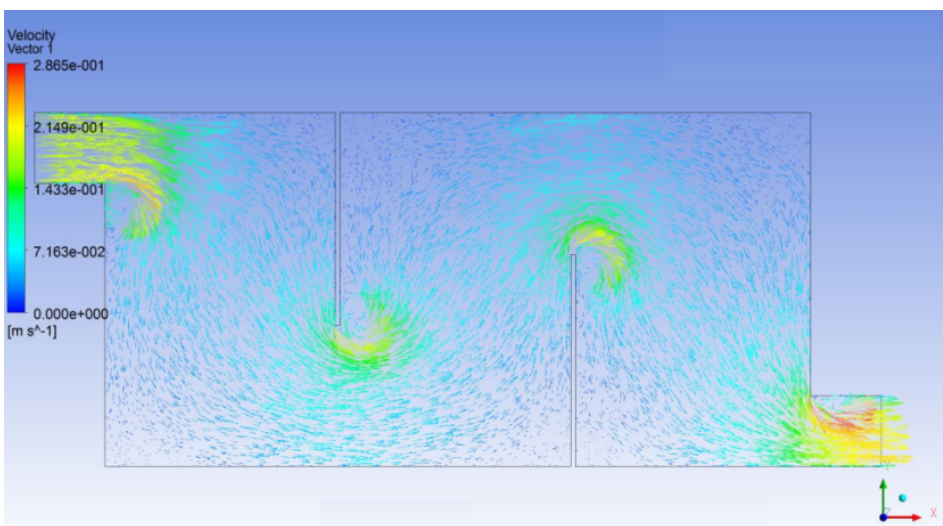

c) 0.6 


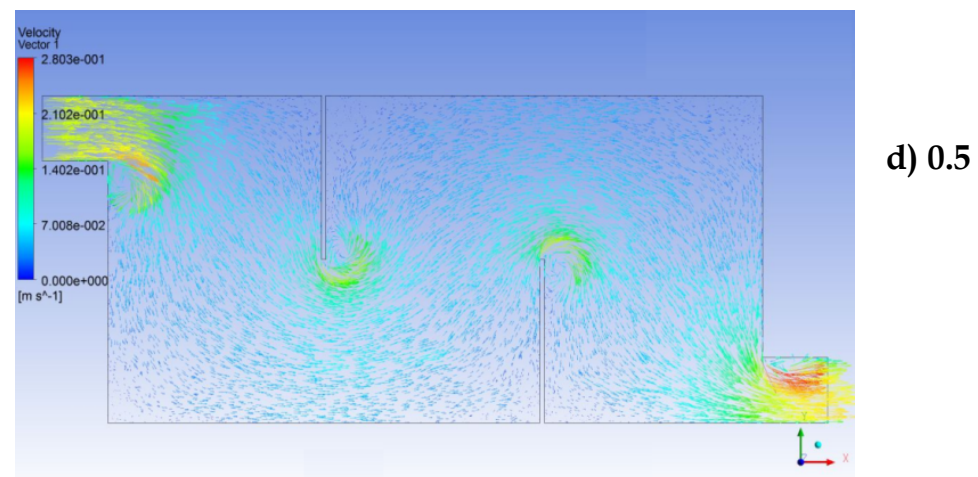

Fig. 3: Flow patterns at various a/ $\mathrm{B}$ ratio $(\mathrm{L} / \mathrm{B}=2, \mathrm{n}=2)$

In Fig.3a $(n=2, a / B=0.8)$ the entry flow extends to the full width of the enclosure and bottom wall has an influence along which the flow is dragged. Due to this, the turning of the flow at the tip of the plate is not sharp and the width of the recirculation region at the tip is wider compared to those at other values of a/B (Figs.3b to d). A similar feature is seen at the top tip of the second baffle. The flow is guided around these tip recirculation regions and the turning angle is decided by the width of these recirculation zones. Separated regions of flow occur on the downstream faces of both the plates. There are large regions of low velocities and weak vortical flow is seen at the corner at the bottom of the front of the second baffle. When the plate length decreases to 0.7 (Fig.3b), the influence of the bottom wall of the enclosure on the tip flow still exists though to a lesser extent compared to the earlier case. The tip recirculation region is smaller and other features are as in the earlier case. With further decrease in a/ B ratio (Figs. $3 c$ and d) there is little influence of the bottom surface of the enclosure on the flow turning at the tips. Due to this the size of the tip vortex region becomes smaller and weaker (as can be made out by the color code of the velocity vectors). The vortical regions at the corners increase in size. As the spacing between the baffles is large, the tip flows are seen to be unaffected by each other. 
When the number of baffles increase to 4 (Fig.4), the features noticed compared to $n=2$ case (Fig.3) are, 1) the tip vortex regions become sharper (except for $\mathrm{a} / \mathrm{B}=0.8$ ), 2) there is flow along the baffles after the flow turning around the tips, 3) recirculating/stagnant regions at the corners reduce, 4) even for $\mathrm{a} / \mathrm{B}=0.6$ and 0.5 (Figs.4c and d), the tip flows at successive baffles appear to be nearly independent of each other.

The situation changes considerably for $n=6$ and 8 , Figs. 5 and 6 . The influence of the relative length of the baffles on the flow field become marked. At a/ $\mathrm{B}=0.8$ and 0.7 , there is a stronger flow along the front faces of all the baffles with the possibility of sharper velocity gradients in the vicinity of the baffles, specially for $n=8$. The flow takes a sharp turn at the tips. A Coanda-like effect appears to influence the sharp turn (similar phenomenon occurs for $\mathrm{n}=2$ and 4). This also gives rise to the possibility of lateral forces on the baffles. There is a marked change between $n=0.7$ and $0.6-$ for 0.6 , the turning flow at the tips are connected giving rise to a sinusoidal or snake-like flow. This feature becomes much more evident at $\mathrm{a} / \mathrm{B}=0.5$ for both $\mathrm{n}=6$ and 8 (Figs.5d and $6 \mathrm{~d}$ ). In both these cases, at $\mathrm{a} / \mathrm{B}=0.8$ and 0.7 , there is a tendency towards plug like flow between the baffles.

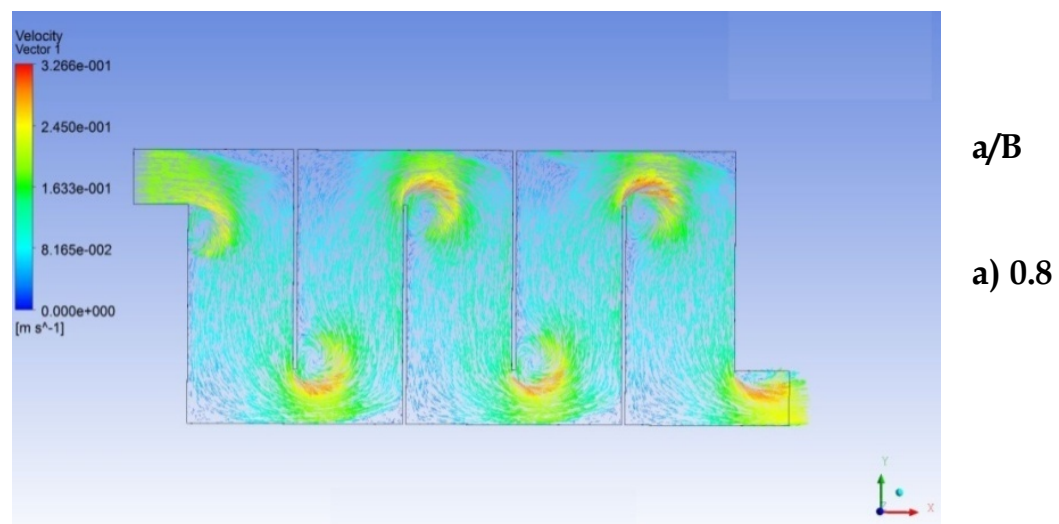



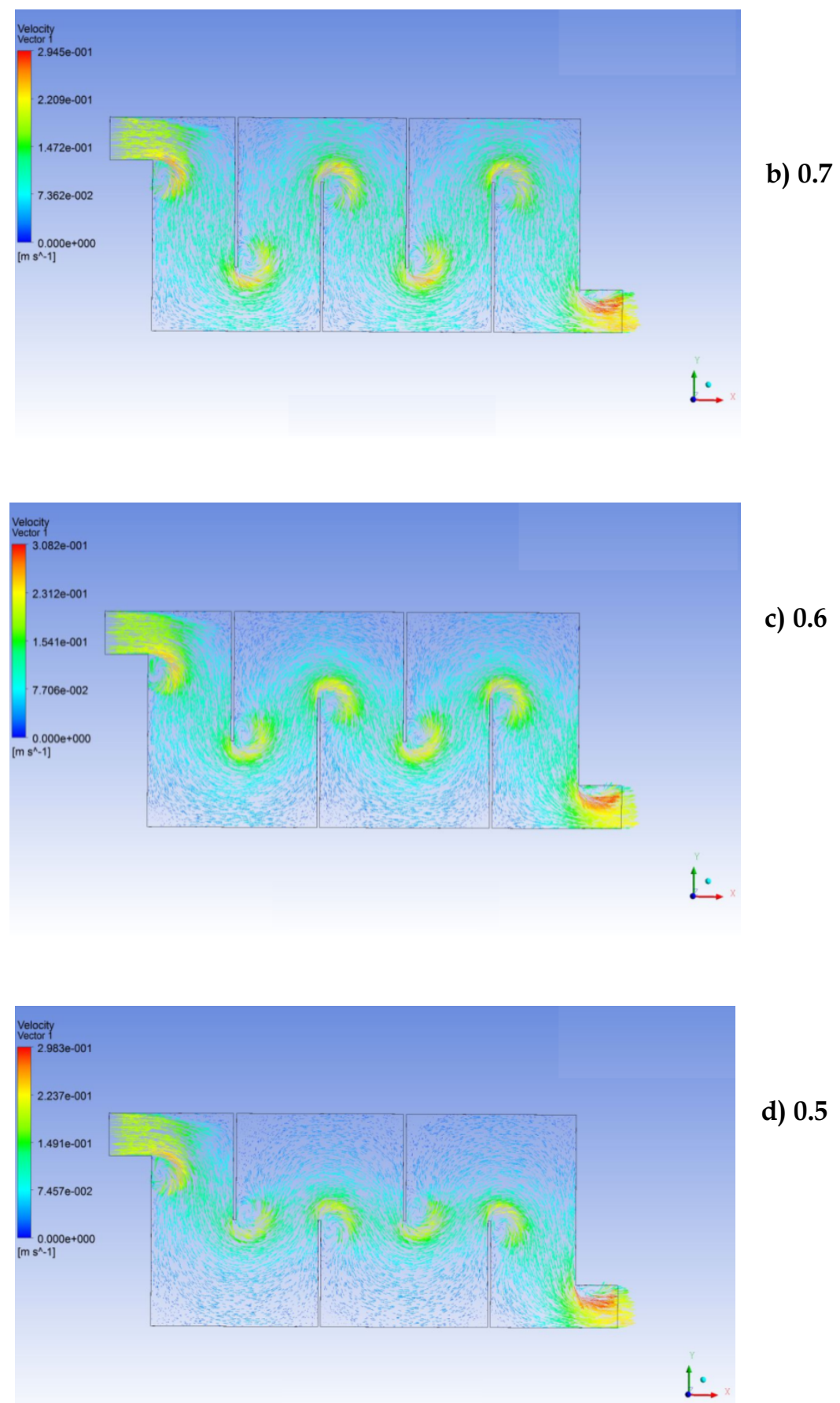

Fig 4: Flow patterns at various a/B ratio $(\mathrm{L} / \mathrm{B}=2, \mathrm{n}=4)$ 


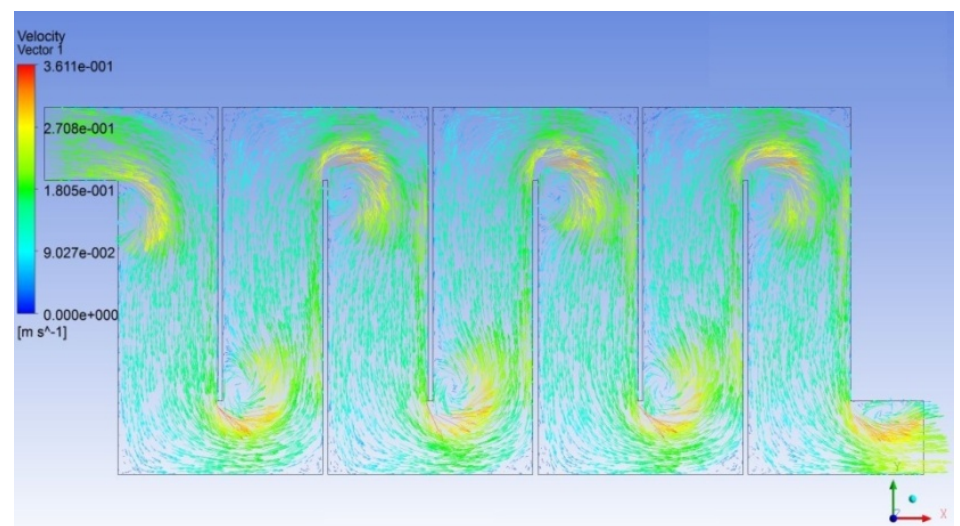

a) 0.8

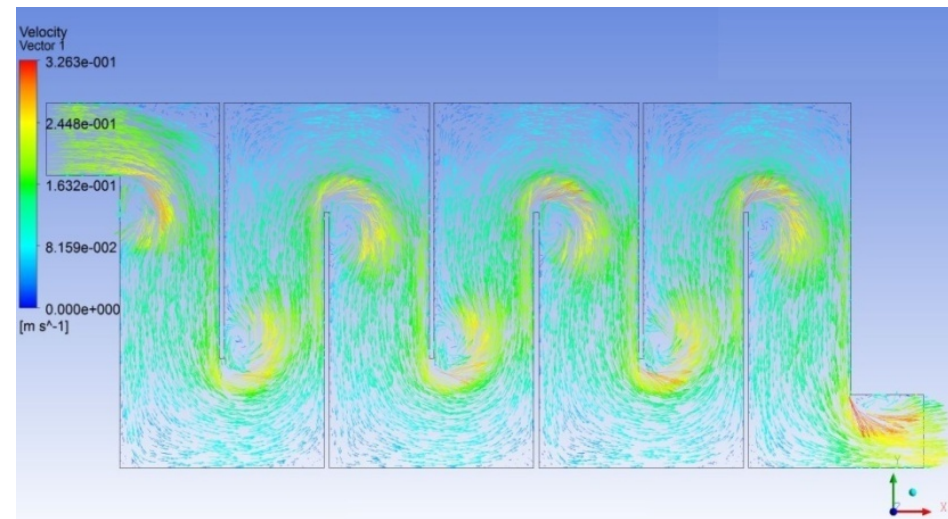

b) 0.7

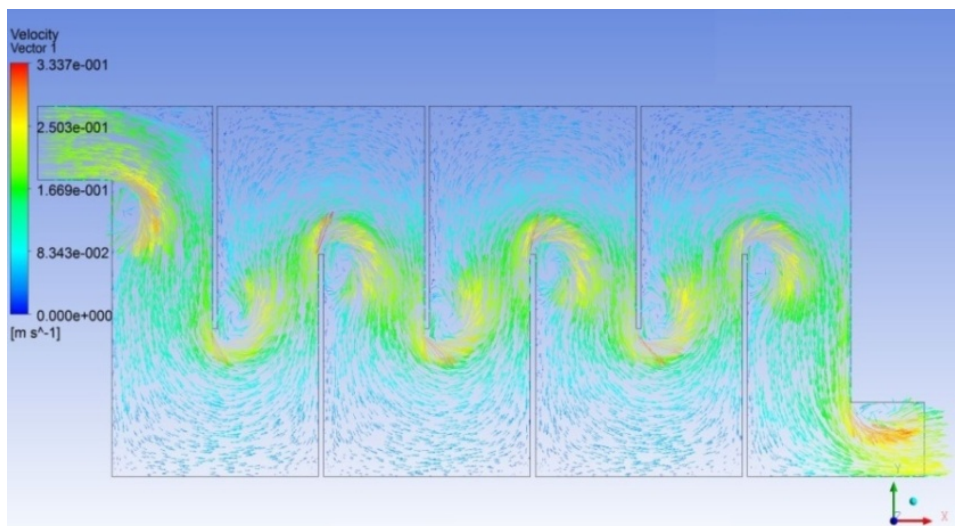

c) 0.6 


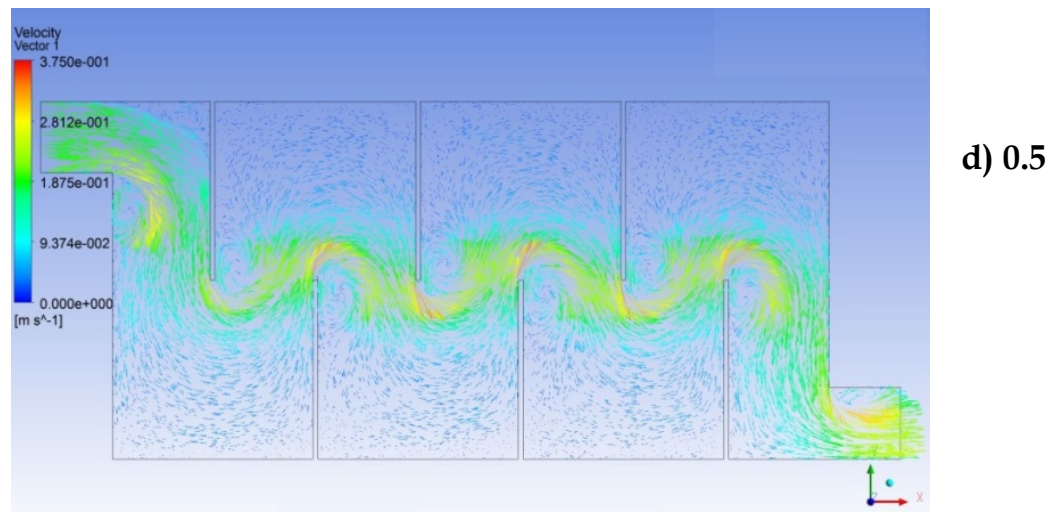

Fig 5: Flow patterns at various a/B ratio $(\mathrm{L} / \mathrm{B}=2, \mathrm{n}=6)$

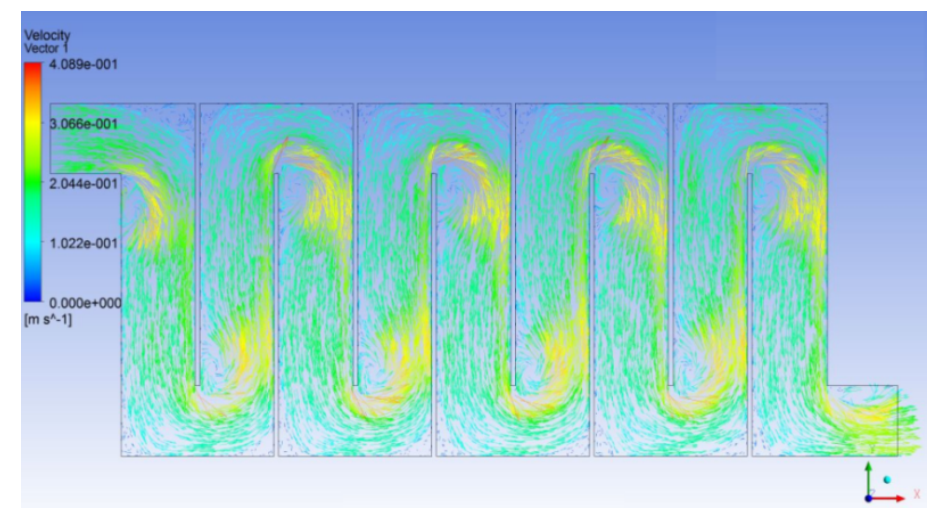

a) 0.8

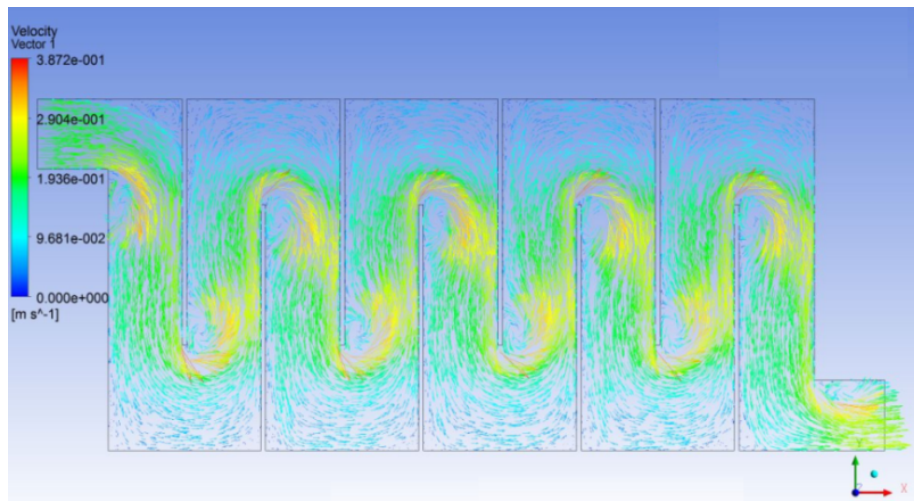

b) 0.7 


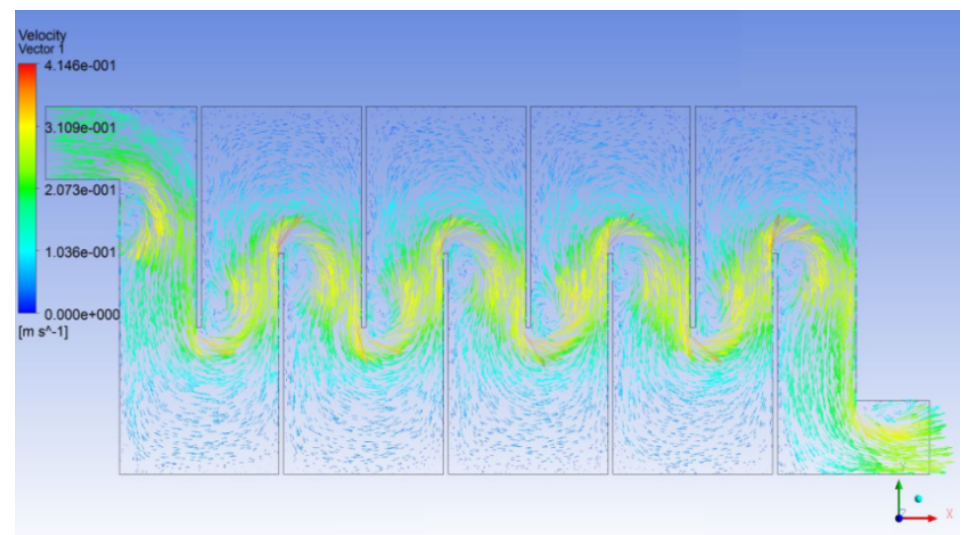

c) 0.6

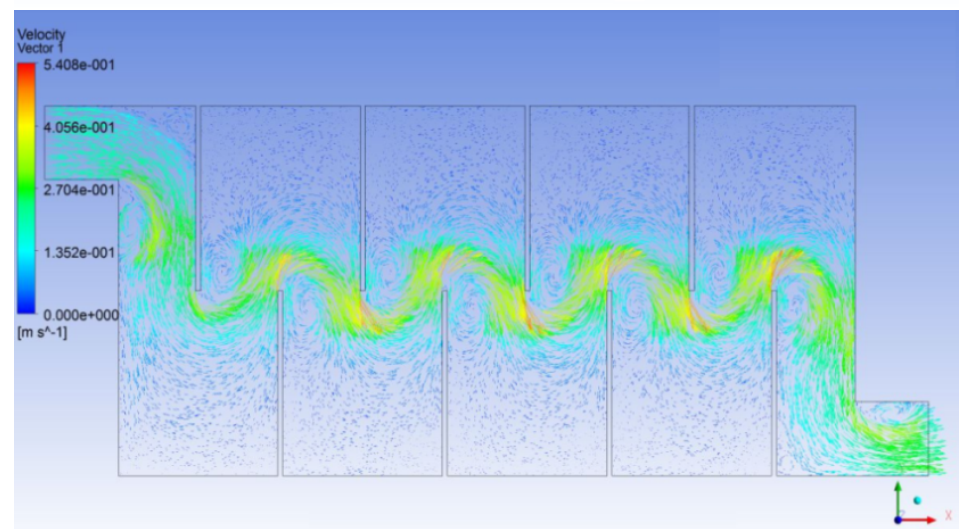

d) 0.5

Fig 6: Flow patterns at various $\mathrm{a} / \mathrm{B}$ ratio $(\mathrm{L} / \mathrm{B}=2, \mathrm{n}=8)$

\section{Concluding Remarks}

Apart from the number of baffles, the ratio a/B appears to have the most significant effect on the flow field. The length of the path of the fluid, the extent of the regions of separation, recirculation and stagnation zones are determined by the a/B ratio. For example, the maximum extent of the stagnation zones occur for the lowest value of a/B (0.5 tested). Another significant effect of the length ratio of the baffle plate i.e. a/B is in the possibility of controlling heat transfer rates, turbulence levels and velocity gradients. A shorter length of the plates may lead to larger regions with temperature and velocity gradients. Hence the a/B ratio plays an important role in controlling the flow. Particularly, when a narrow 
region of high velocity with increased turbulence is required in front i.e., upstream of a tube bundle, plates with lower values of $\mathrm{a} / \mathrm{B}$ are preferable. Opposed to this, when it is required to have a long path of the flow with high retention time, as required in flocculation process tanks, baffles with longer length i.e. high a/B values may be desirable.

\section{Acknowledgement}

This work is carried out as a part of the VTU Project No.VTU / Aca. / 2009-10. The financial grant is gratefully acknowledged. The authors express their sincere thanks to the Management of BTL IT for their support and encouragement.

\section{References}

[1] L C Demartini, H A Vielmo and S V Moller, Numeric and experimental analysis of the turbulent flow through a channel with baffle plates, $J$. Brazilian Society of Mechanical Sciences and Engineering, vol. 26, pp. 153-169, 2004.

[2] M T Al-Atabi, S B Chin, S Al- Zuhair and X Y Luo, Pressure drop in laminar and turbulent flows in circular pipe with baffles-An experimental and analytical study, Int. J. Fluid Mechanics, vol. 33, 319, 2006.

[3] C Gualtieri, Analysis of the effect of baffles number on a contact tank efficiency with Multiphysics 3.3, Excerpt from the Proceedings of the COMSOL Users Conference Grenoble, 2007.

[4] S K Oh, A B K Putra and S W Ahn, Heat transfer and frictional characteristics in rectangular channel with inclined perforated baffles, Proceedings of World Academy of Science, Engineering and Technology, vol. 37, pp. 324-329, 2009. 